\begin{tabular}{|c|c|}
\hline Title & Improved Finite Element Formulation in Terms of the Magnetic Field V ector for Dielectric Waveguides \\
\hline Author(s) & Koshiba, M.; Hay ata, K.; Suzuki, M. \\
\hline Citation & IEEE Transactions on Microwave Theory and Techniques, 33(3), 227-233 \\
\hline Issue Date & $1985-03$ \\
\hline Doc URL & http:/hdl. handle.net/2115/6042 \\
\hline Rights & $\begin{array}{l}\text { O1985 IEEE. Personal use of this material is permitted. However, permission to reprint/republish this material for } \\
\text { advertising or promotional purposes or for creating new collective works for resale or redistribution to servers or lists, } \\
\text { or to reuse any copyrighted component of this work in other works must be obtained from the IEEE. } \\
\text { IEEE, IEEE Transactions on Microwave Theory and Techniques, 33(3), 1985, p227-233 }\end{array}$ \\
\hline Type & article \\
\hline File Information & ITMTT33_3.pdf \\
\hline
\end{tabular}

Instructions for use 


\title{
Improved Finite-Element Formulation in Terms of the Magnetic Field Vector for Dielectric Waveguides
}

\author{
MASANORI KOSHIBA, SENIOR MEMBER, IEEE, KAZUYA HAYATA, \\ AND MICHIO SUZUKI, SENIOR MEMBER, IEEE
}

\begin{abstract}
An improved finite-element method for the analysis of dielectric waveguides is formulated in terms of all three components of the magnetic field $\boldsymbol{H}$. In this approach, the spurious, nonphysical solutions do not appear anywhere above the "air-line," and therefore the present formulation is very useful for the analysis of the surface-wave modes of dielectric waveguides. The application of this improved finite-element method to the dielectric waveguides with perfect electric and magnetic conductors is also discussed. In particular, the discussion is how to use the conditions on a boundary surface of a perfect electric or magnetic conductor whose normal direction is not coincident with the direction of a coordinate axis. Application of these boundary conditions for perfect conductors to the dielectric waveguides with planes of symmetry reduces the matrix size. The strength of this approach to boundary conditions is not just the economical use of computer memory but the elimination of spurious solutions through rigorous enforcement of boundary conditions as rell.
\end{abstract}

\section{INTRODUCTION}

S EVERAL METHODS for the analysis of dielectric waveguides in Fig. 1 have been proposed, and the vectorial finite-element formulation in terms of the longitudinal electric $\left(E_{z}\right)$ and magnetic $\left(H_{z}\right)$ field components, which enables one to compute accurately the mode spectrum of a waveguide with arbitrary cross section, is widely used [1]-[14]. The most serious difficulty in using the finite-element analysis, for inhomogeneous dielectric waveguides, is the appearance of the so-called spurious, nonphysical solutions [1]-[14]. The longitudinal $E_{z}-H_{z}$ formulation contains mathematical singularities [2], [3]. Recently, Mabaya, Lagasse, and Vandenbulcke [12] found that by explicitly enforcing the continuity of the tangential components of the transversal fields, at the interface, by means of Lagrange multipliers, most of the spurious solutions disappear. The disadvantage of this method lies in the greatly increased complexity of the program and of the numerical operators that have to be used to enforce those continuity conditions [12]. Konrad [15] proposed the vectorial finite-element formulation in terms of all three components $\left(H_{x}, H_{y}\right.$, and $\left.H_{z}\right)$ of the magnetic field $\boldsymbol{H}$. The three-component formulation does not contain mathematical singularities as is the case with the $E_{z}-H_{z}$ formulation, but the spurious solutions do appear [15]-[17]. As

Manuscript received May 30, 1984; revised October 15, 1984.

The authors are with the Department of Electronic Engineering, Hokkaido University, Sapporo, 060, Japan noted by Davies, Fernandez, and Philippou [16], the spurious solutions in the three-component formulation do not satisfy the divergence relation for $\boldsymbol{H}, \nabla \cdot \boldsymbol{H}=0$.

In this paper, an improved finite-element method for the analysis of dielectric waveguides is formulated in terms of all three components of $\boldsymbol{H}$. For an abrupt discontinuity in the permittivity in an inhomogeneous medium, there is an abrupt change in the electric field $\boldsymbol{E}$. In such cases, it is advantageous to solve for the values of $\boldsymbol{H}$ at the nodal points. In this approach, the spurious solutions do not appear anywhere above the "air-line" corresponding to $\beta / k_{0}=1$ in a $\beta / k_{0}$ versus $k_{0}$ diagram (a plot of $\beta / k_{0}$ on the vertical axis against $k_{0}$ on the horizontal axis), where $k_{0}$ is the wavenumber of free space and $\beta$ is the phase constant in the $z$-direction. Therefore, the present formulation is very useful for the analysis of the surface-wave modes of dielectric waveguides which correspond to the solutions above the "air-line."

The application of this improved finite-element method to the dielectric waveguides with perfect electric and magnetic conductors is also discussed. In particular, the discussion is how to use the conditions on a boundary surface of a perfect electric or magnetic conductor whose normal direction is not coincident with the direction of a coordinate axis. In the analysis of dielectric waveguides with planes of symmetry, these boundary conditions for perfect conductors are used on each plane of symmetry. Application of these conditions reduces the matrix size. The strength of this approach to boundary conditions is not just the economical use of computer memory but the elimination of spurious solutions through rigorous enforcement of boundary conditions as well.

\section{Functional Formulation}

We consider a dielectric waveguide with arbitrary crosssection $\Omega$ in the $x y$-plane as shown in Fig. 1 . With a time dependence of the form $\exp (j \omega t)$ being implied, Maxwell's equations are

$$
\begin{aligned}
\nabla \times \boldsymbol{E} & =-j \omega \mu_{0} \boldsymbol{H} \\
\nabla \times \boldsymbol{H} & =j \omega \epsilon_{0}[K] \boldsymbol{E}
\end{aligned}
$$

where $\omega$ is the angular frequency, $\epsilon_{0}$ and $\mu_{0}$ are the permittivity and permeability of free space, respectively, 


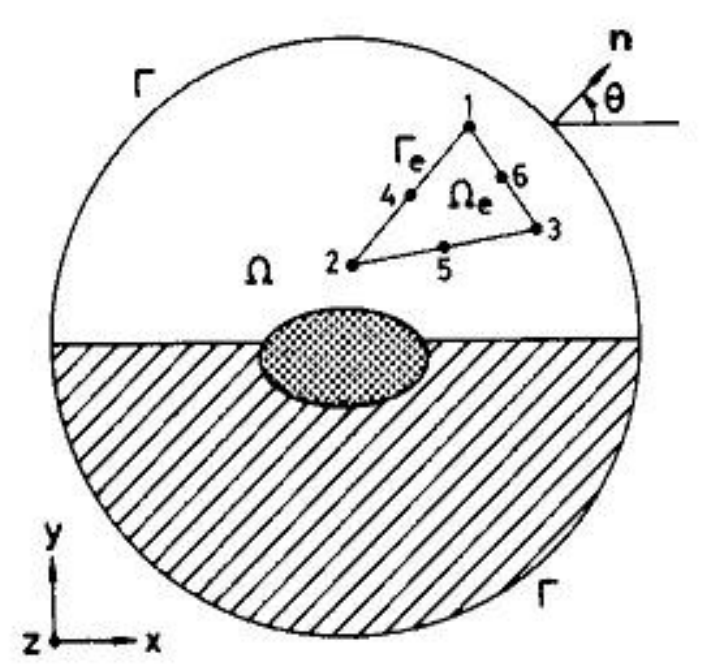

Fig. 1. Geometry of the problem.

$[K]$ is the relative permittivity tensor, and $[\cdot]$ denotes a matrix.

By substituting (2) into (1), the following wave equation is derived:

$$
\nabla \times\left([K]^{-1} \nabla \times \boldsymbol{H}\right)-k_{0}^{2} \boldsymbol{H}=0
$$

where

$$
k_{0}^{2}=\omega^{2} \epsilon_{0} \mu_{0} .
$$

The functional [15] for (3) is known to be

$$
F=\iint_{\Omega}\left[(\nabla \times \boldsymbol{H})^{*} \cdot\left([K]^{-1} \nabla \times \boldsymbol{H}\right)-k_{0}^{2} \boldsymbol{H}^{*} \cdot \boldsymbol{H}\right] d \Omega
$$

where the asterisk denotes a complex conjugate. The formulation of (5) does not contain mathematical singularities as is the case with the $E_{z}-H_{z}$ formulation, but the spurious solutions do appear [15]-[17]. These spurious solutions fall into two fairly clear categories [16]. The first one $\left(S_{1}\right)$ can be characterized as follows:

$$
\nabla \times \boldsymbol{H}=0, \quad \nabla \cdot \boldsymbol{H} \neq 0 \quad \text { for } k_{0}^{2}=0 .
$$

The second group $\left(S_{2}\right)$ can be characterized as follows:

$$
\nabla \times \boldsymbol{H} \neq 0, \quad \nabla \cdot \boldsymbol{H} \neq 0 \quad \text { for } k_{0}^{2}>0 .
$$

These spurious solutions do not satisfy the relation $\nabla \cdot \boldsymbol{H}=0$ [16], [17].

Now, we consider the following functional [18]-[20]:

$$
\tilde{F}=F+\iint_{\Omega}(\nabla \cdot \boldsymbol{H})^{*}(\nabla \cdot \boldsymbol{H}) d \Omega
$$

For the functional (8), the first variation $\delta \tilde{F}$ is given by

$$
\begin{aligned}
\delta \tilde{F}= & \iint_{\Omega} \delta \boldsymbol{H}^{*} \\
& \cdot\left[\nabla \times\left([K]^{-1} \nabla \times \boldsymbol{H}\right)-\nabla(\nabla \cdot \boldsymbol{H})-k_{0}^{2} \boldsymbol{H}\right] d \Omega \\
& -\int_{\Gamma} \delta \boldsymbol{H}^{*} \cdot\left[\boldsymbol{n} \times\left([K]^{-1} \nabla \times \boldsymbol{H}\right)-\boldsymbol{n}(\nabla \cdot \boldsymbol{H})\right] d \Gamma
\end{aligned}
$$

where $\Gamma$ represents the contour of the region $\Omega, n$ is the outward unit normal vector to $\Gamma$, and the term $n \times$ $\left([K]^{-1} \nabla \times \boldsymbol{H}\right)$ corresponds to the tangential components of the electric field $\boldsymbol{E}$ on $\Gamma$. The stationarity requirement $\delta \tilde{F}=0$ shows that

$$
\nabla \times\left([K]^{-1} \nabla \times \boldsymbol{H}\right)-\nabla(\nabla \cdot \boldsymbol{H})-k_{0}^{2} \boldsymbol{H}=0
$$

as the Euler equation and

$$
\begin{aligned}
\boldsymbol{n} \times\left([K]^{-1} \nabla \times \boldsymbol{H}\right) & =0 \\
\boldsymbol{n}(\nabla \cdot \boldsymbol{H}) & =0
\end{aligned}
$$

on perfect electric conductor

on perfect magnetic conductor

as natural boundary conditions, since $\delta \boldsymbol{H}^{*}$ in (9) is arbj. trary.

Multiplying (10a) by $\boldsymbol{H}^{*}$ and integrating over the region $\Omega$, the following equation is obtained using Green's for. mula and the boundary conditions on $\Gamma$ :

$$
\begin{array}{r}
\iint_{\Omega}\left[(\nabla \times \boldsymbol{H})^{*} \cdot\left([K]^{-1} \nabla \times \boldsymbol{H}\right)+(\nabla \cdot \boldsymbol{H})^{*}(\nabla \cdot \boldsymbol{H})\right. \\
\left.\left.-k_{0}^{2} \boldsymbol{H}^{*} \cdot \boldsymbol{H}\right)\right] d \Omega=0 .
\end{array}
$$

In (11), if $[K]^{-1}$ is a positive definite matrix, then $\nabla \times H$ $=0$ and $\nabla \cdot \boldsymbol{H}=0$ are satisfied for $k_{0}^{2}=0$. Therefore, the spurious solutions $S_{1}$ are eliminated.

Taking divergence of (10a), we obtain

$$
\left(\nabla^{2}+k_{0}^{2}\right)(\nabla \cdot \boldsymbol{H})=0 .
$$

If the curl of $\boldsymbol{H}$ is not zero for $k_{0}^{2}>0$, the eigenvalues $k_{0}^{2}$ of (10) cannot satisfy (12). Therefore, the eigenvectors of (10) should obey $\nabla \cdot \boldsymbol{H}=0$ and the spurious solutions $S_{2}$ are eliminated.

When $\nabla \times \boldsymbol{H}=0$ for $k_{0}^{2}>0,(8)$ may have the solutions other than those of (3) [19]. This new group $\left(S_{3}\right)$ char. acterized by

$$
\nabla \times \boldsymbol{H}=0, \quad \nabla \cdot \boldsymbol{H} \neq 0 \quad \text { for } k_{0}^{2}>0
$$

obeys the following equations:

$$
\begin{aligned}
\boldsymbol{H} & =\nabla \phi & & \\
\left(\nabla^{2}+k_{0}^{2}\right) \phi & =0 & & \text { in region } \Omega \\
\partial \phi / \partial n & =0 & & \text { on perfect electric conductor } \\
\phi & =0 & & \text { on perfect magnetic conductor }
\end{aligned}
$$

where $\phi$ is the scalar field. The magnetic field $\boldsymbol{H}$ of (14) satisfies the stationarity requirement $\delta \tilde{F}=0$, but the divergence of $\boldsymbol{H}$ is not zero. Therefore, in the finite-element analysis using (8), the spurious solution $S_{3}$ which are not included in (5) do appear. Fortunately, the solutions $S_{3}$ are equivalent to the TE modes of "hollow" waveguides (replace $\phi$ in $(14 \mathrm{~b})-(14 \mathrm{~d})$ with $H_{z}$ ) and the appearance is limited to the region $\beta / k_{0}<1$. They do not appear any. where above the "air-line." Therefore, if one is interested only in the solutions in the region $\beta / k_{0} \geqslant 1$ which correspond to the surface-wave modes of dielectric waveguides [4]-[7], [10]-[13], [17], [21], [22], the appearance of the solutions $S_{3}$ is not a serious problem. The value of (14) is that it enables us to evaluate the behavior of the spurious solutions $S_{3}$ of the finite-element method based on (8). On the other hand, the spurious solutions $S_{1}$ and $S_{2}$ of the finite-element method based on (5) are unpredictable.

\section{Finite-Element Discretization}

Dividing the cross-section $\Omega$ of the waveguide with a diagonal permittivity tensor into a number of second-order 
triangular elements in Fig. 1, the magnetic fields within each element are defined in terms of the magnetic fields at the corner and midside nodal points:

$$
\boldsymbol{H}=[N]^{T}\{H\}_{e} \exp (-j \beta z)
$$

where

$$
\begin{aligned}
& \{H\}_{e}=\left[\begin{array}{l}
\left\{H_{x}\right\}_{e} \\
\left\{H_{y}\right\}_{e} \\
\left\{H_{z}\right\}_{e}
\end{array}\right] \\
& {[N]=\left[\begin{array}{ccc}
\{N\} & \{0\} & \{0\} \\
\{0\} & \{N\} & \{0\} \\
\{0\} & \{0\} & j\{N\}
\end{array}\right]} \\
& \{N\}=\left[\begin{array}{llllll}
N_{1} & N_{2} & N_{3} & N_{4} & N_{5} & N_{6}
\end{array}\right]^{T} \text {. }
\end{aligned}
$$

Here $\left\{H_{x}\right\}_{e},\left\{H_{y}\right\}_{e}$, and $\left\{H_{z}\right\}_{e}$ are magnetic field vectors corresponding to the nodal points within each element, $\{0\}$ is a null vector, $T,\{\cdot\}$, and $\{\cdot\}^{T}$ denote a transpose, a column vector, and a row vector, respectively, and the shape functions $N_{1}$ to $N_{6}$ are given by

$$
\begin{aligned}
& N_{1}=L_{1}\left(2 L_{1}-1\right) \\
& N_{2}=L_{2}\left(2 L_{2}-1\right) \\
& N_{3}=L_{3}\left(2 L_{3}-1\right) \\
& N_{4}=4 L_{1} L_{2} \\
& N_{5}=4 L_{2} L_{3} \\
& N_{6}=4 L_{3} L_{1}
\end{aligned}
$$

with the area coordinates $L_{1}, L_{2}$, and $L_{3}$ [4], [10]. The relation equation between the area coordinates and Cartesian coordinates is given by

$$
\left[\begin{array}{l}
x \\
y \\
1
\end{array}\right]=\left[\begin{array}{ccc}
x_{1} & x_{2} & x_{3} \\
y_{1} & y_{2} & y_{3} \\
1 & 1 & 1
\end{array}\right]\left[\begin{array}{l}
L_{1} \\
L_{2} \\
L_{3}
\end{array}\right]
$$

where

$$
\begin{aligned}
{[S]_{e} } & =\iint_{e}[B]^{*}[K]_{e}^{-1}[B]^{T} d x d y \\
{[T]_{e} } & =\iint_{e}[N]^{*}[N]^{T} d x d y \\
{[U]_{e} } & =\iint_{e}\{C\}\{C\}^{T} d x d y \\
{[B] } & =\left[\begin{array}{ccc}
\{0\} & -j \beta\{N\} & -\partial\{N\} / \partial y \\
j \beta\{N\} & \{0\} & \partial\{N\} / \partial x \\
j \partial\{N\} / \partial y & -j \partial\{N\} / \partial x & \{0\}
\end{array}\right] \\
\{C\} & =\left[\begin{array}{c}
\partial\{N\} / \partial x \\
\partial\{N\} / \partial y \\
\beta\{N\}
\end{array}\right] .
\end{aligned}
$$

The functional for the whole region $\Omega$ is given by

$$
\tilde{F}=\{H\}^{T}\left([S]+[U]-k_{0}^{2}[T]\right)\{H\}
$$

where $\{H\}$ is the nodal magnetic field vector and, for loss-free media, $[S],[T]$, and $[U]$ are real, symmetric matrices. Variation of (27) with respect to the nodal variables leads to the following eigenvalue problem:

$$
([S]+[U])\{H\}-k_{0}^{2}[T]\{H\}=\{0\} .
$$

Using the functional (5), we obtain the following eigenvalue problem:

$$
[S]\{H\}-k_{0}^{2}[T]\{H\}=\{0\}
$$

\section{BOUNDARY CONDITIONS}

In (27), the nodal magnetic field vector $\{H\}$ should be forced to satisfy the boundary conditions on $\Gamma$ in Fig. 1, where the unit vector $\boldsymbol{n}$ normal to $\Gamma$ lies at an angle $\theta$ from the $x$-axis in the $x y$-plane. The functional (27) can be rewritten as

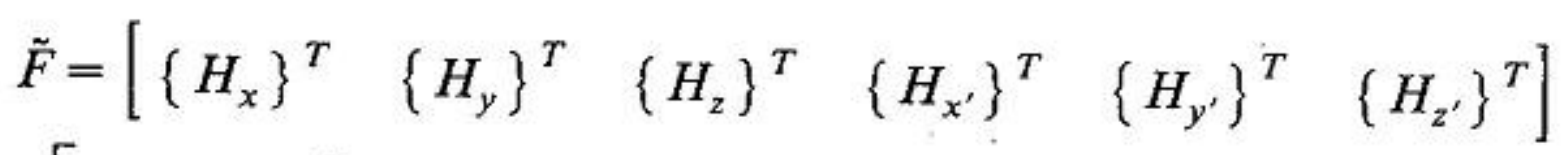

$$
\begin{aligned}
& {\left[\begin{array}{cccccc}
{\left[A_{x x}\right]} & {\left[A_{x y}\right]} & {\left[A_{x z}\right]} & {\left[A_{x x^{\prime}}\right]} & {\left[A_{x y^{\prime}}\right]} & {\left[A_{x z^{\prime}}\right]} \\
{\left[A_{y x}\right]} & {\left[A_{y y}\right]} & {\left[A_{y z}\right]} & {\left[A_{y x^{\prime}}\right]} & {\left[A_{y y^{\prime}}\right]} & {\left[A_{y z^{\prime}}\right]} \\
{\left[A_{z x}\right]} & {\left[A_{z y}\right]} & {\left[A_{z z}\right]} & {\left[A_{z x^{\prime}}\right]} & {\left[A_{z y^{\prime}}\right]} & {\left[A_{z z^{\prime}}\right]} \\
{\left[A_{x^{\prime} x}\right]} & {\left[A_{x^{\prime} y}\right]} & {\left[A_{x^{\prime} z}\right]} & {\left[A_{x^{\prime} x^{\prime}}\right]} & {\left[A_{x^{\prime} y^{\prime}}\right]} & {\left[A_{x^{\prime} z^{\prime}}\right]} \\
{\left[A_{y^{\prime} x}\right]} & {\left[A_{y^{\prime} y}\right]} & {\left[A_{y^{\prime} z}\right]} & {\left[A_{y^{\prime} x^{\prime}}\right]} & {\left[A_{y^{\prime} y^{\prime}}\right]} & {\left[A_{y^{\prime} z^{\prime}}\right]} \\
{\left[A_{z^{\prime} x}\right]} & {\left[A_{z^{\prime} y}\right]} & {\left[A_{z^{\prime} z}\right]} & {\left[A_{z^{\prime} x^{\prime}}\right]} & {\left[A_{z^{\prime} y^{\prime}}\right]} & {\left[A_{z^{\prime} z^{\prime}}\right]}
\end{array}\right]\left[\begin{array}{l}
\left\{H_{x}\right\} \\
\left\{H_{y}\right\} \\
\left\{H_{z}\right\} \\
\left\{H_{x^{\prime}}\right\} \\
\left\{H_{y^{\prime}}\right\} \\
\left\{H_{z^{\prime}}\right\}
\end{array}\right]}
\end{aligned}
$$

where $\left(x_{k}, y_{k}\right)$ are the Cartesian coordinates of the vertex $k(k=1,2,3)$ of the triangle.

Substituting (15) into the functional (8), for each element we obtain

$\tilde{F}_{e}=\{H\}_{e}^{T}[S]_{e}\{H\}_{e}+\{H\}_{e}^{T}[U]_{e}\{H\}_{e}$

$$
-k_{0}^{2}\{H\}_{e}^{T}[T]_{e}\{H\}_{e}
$$

where the components of the $\left\{H_{i}\right\}$ vector are the values of the magnetic field $H_{i}$ at all nodal points in $\Omega$ except $\Gamma$, the components of the $\left\{H_{i^{\prime}}\right\}$ vector are the values of $H_{i}$ at all nodal points on $\Gamma$, and $\left[A_{x x}\right],\left[A_{x y}\right], \cdots$, and $\left[A_{z^{\prime} z^{\prime}}\right]$ are the submatrices of $[A] \equiv[S]+[U]-k_{0}^{2}[T]$.

Using the boundary condition for the perfect electric conductor $\boldsymbol{n} \cdot \boldsymbol{H}=0$, namely

$$
\left\{H_{x^{\prime}}\right\}=-\tan \theta\left\{H_{y^{\prime}}\right\}
$$


on $\Gamma$ and minimizing (30), we obtain

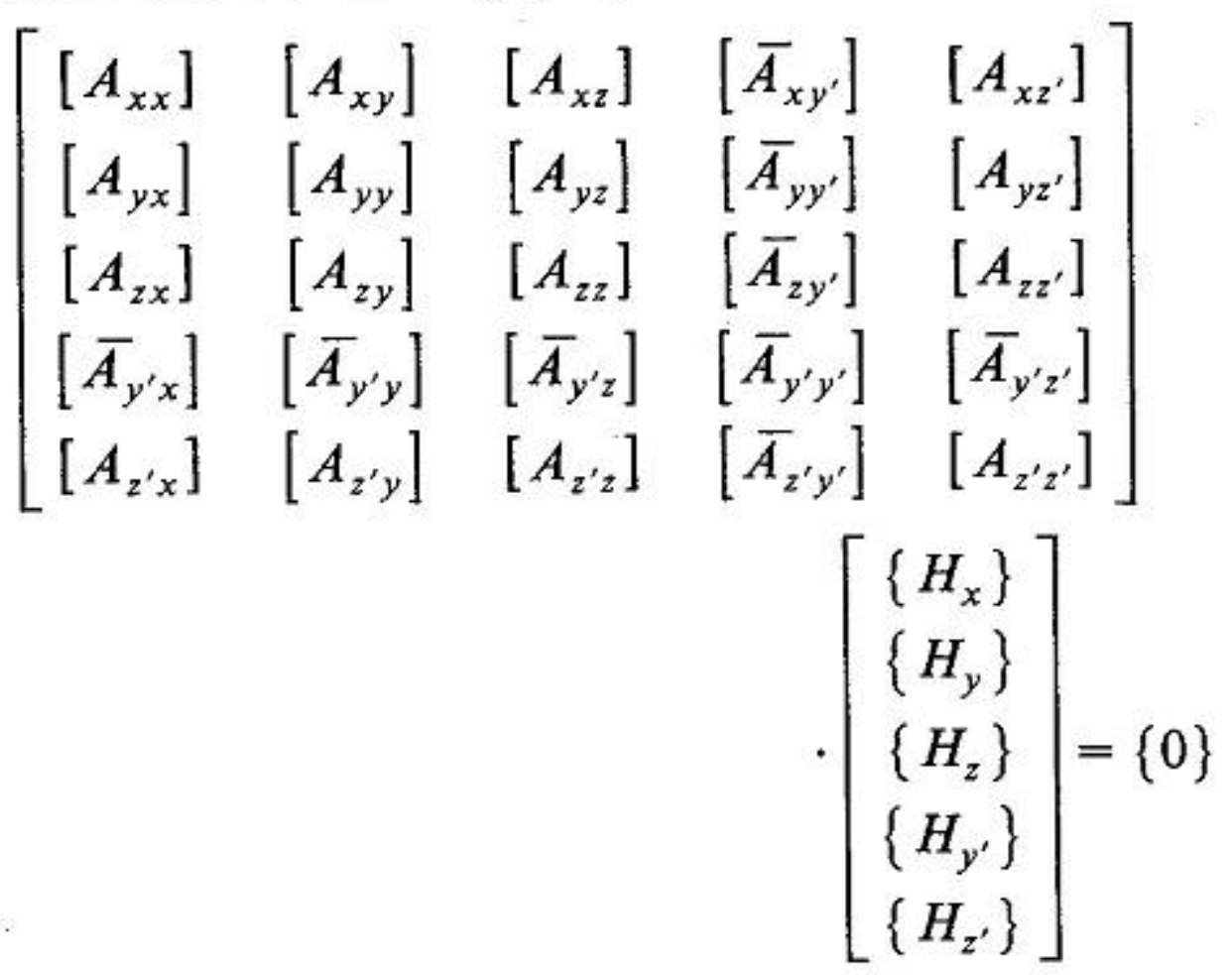

where

$$
\begin{aligned}
& {\left[\bar{A}_{y^{\prime} y^{\prime}}\right]=\left[A_{y^{\prime} y^{\prime}}\right]-\tan \theta\left(\left[A_{x^{\prime} y^{\prime}}\right]+\left[A_{y^{\prime} x^{\prime}}\right]\right)+\tan ^{2} \theta\left[A_{x^{\prime} x^{\prime}}\right]} \\
& {\left[\bar{A}_{j y^{\prime}}\right]=\left[A_{j y^{\prime}}\right]-\tan \theta\left[A_{j x^{\prime}}\right], \quad j=x, y, z, z^{\prime}} \\
& {\left[\bar{A}_{y^{\prime} j}\right]=\left[A_{y^{\prime} j}\right]-\tan \theta\left[A_{x^{\prime} j}\right], \quad j=x, y, z, z^{\prime} .}
\end{aligned}
$$

Using the boundary condition for the perfect magnetic conductor $\boldsymbol{n} \times \boldsymbol{H}=0$, namely

$$
\begin{aligned}
& \left\{H_{x^{\prime}}\right\}=\cot \theta\left\{H_{y^{\prime}}\right\} \\
& \left\{H_{z^{\prime}}\right\}=\{0\}
\end{aligned}
$$

on $\Gamma$ and minimizing (30), we obtain

$$
\left[\begin{array}{llll}
{\left[A_{x x}\right]} & {\left[A_{x y}\right]} & {\left[A_{x z}\right]} & {\left[\bar{A}_{x y^{\prime}}\right]} \\
{\left[A_{y x}\right]} & {\left[A_{y y}\right]} & {\left[A_{y z}\right]} & {\left[\bar{A}_{y y^{\prime}}\right]} \\
{\left[A_{z x}\right]} & {\left[A_{z y}\right]} & {\left[A_{z z}\right]} & {\left[\bar{A}_{z y^{\prime}}\right]} \\
{\left[\overline{A_{y^{\prime} x}}\right]} & {\left[\bar{A}_{y^{\prime} y}\right]} & {\left[\bar{A}_{y^{\prime} z}\right]} & {\left[\bar{A}_{y^{\prime} y^{\prime}}\right]}
\end{array}\right]\left[\begin{array}{l}
\left\{H_{x}\right\} \\
\left\{H_{y}\right\} \\
\left\{H_{z}\right\} \\
\left\{H_{y^{\prime}}\right\}
\end{array}\right]=\{0\}
$$

where

$$
\begin{aligned}
{\left[\bar{A}_{y^{\prime} y^{\prime}}\right] } & =\left[A_{y^{\prime} y^{\prime}}\right]+\cot \theta\left(\left[A_{x^{\prime} y^{\prime}}\right]+\left[A_{y^{\prime} x^{\prime}}\right]\right)+\cot ^{2} \theta\left[A_{x^{\prime} x^{\prime}}\right] \\
{\left[\overline{A_{j y^{\prime}}}\right] } & =\left[A_{j y^{\prime}}\right]+\cot \theta\left[A_{j x^{\prime}}\right], \quad j=x, y, z \\
{\left[\overline{A_{y^{\prime} j}}\right] } & =\left[A_{y^{\prime} j}\right]+\cot \theta\left[A_{x^{\prime} j}\right], \quad j=x, y, z .
\end{aligned}
$$

When $\tan \theta \rightarrow \infty$ in (33) and $\cot \theta \rightarrow \infty$ in (36), $\left\{H_{y^{\prime}}\right\}$, $\left[\bar{A}_{y^{\prime} y^{\prime}}\right],\left[\bar{A}_{j y^{\prime}}\right]$, and $\left[\bar{A}_{y^{\prime} j}\right]$ should be replaced by $\left\{H_{x^{\prime}}\right\}$, [ $\left.A_{x^{\prime} x^{\prime}}\right],\left[A_{j x^{\prime}}\right]$, and $\left[A_{x^{\prime} j}\right]$, respectively. It should be noted that (32) and (35) can be used to obtain the dispersion characteristics of dielectric waveguides with planes of symmetry.

\section{NUMERICAL Results}

First, let us consider a half-filled dielectric waveguide as shown in Fig. 2, where $n$ is the refractive index. We subdivide one half of the cross section into second-order triangular elements. The solid and dashed lines in Fig. 2

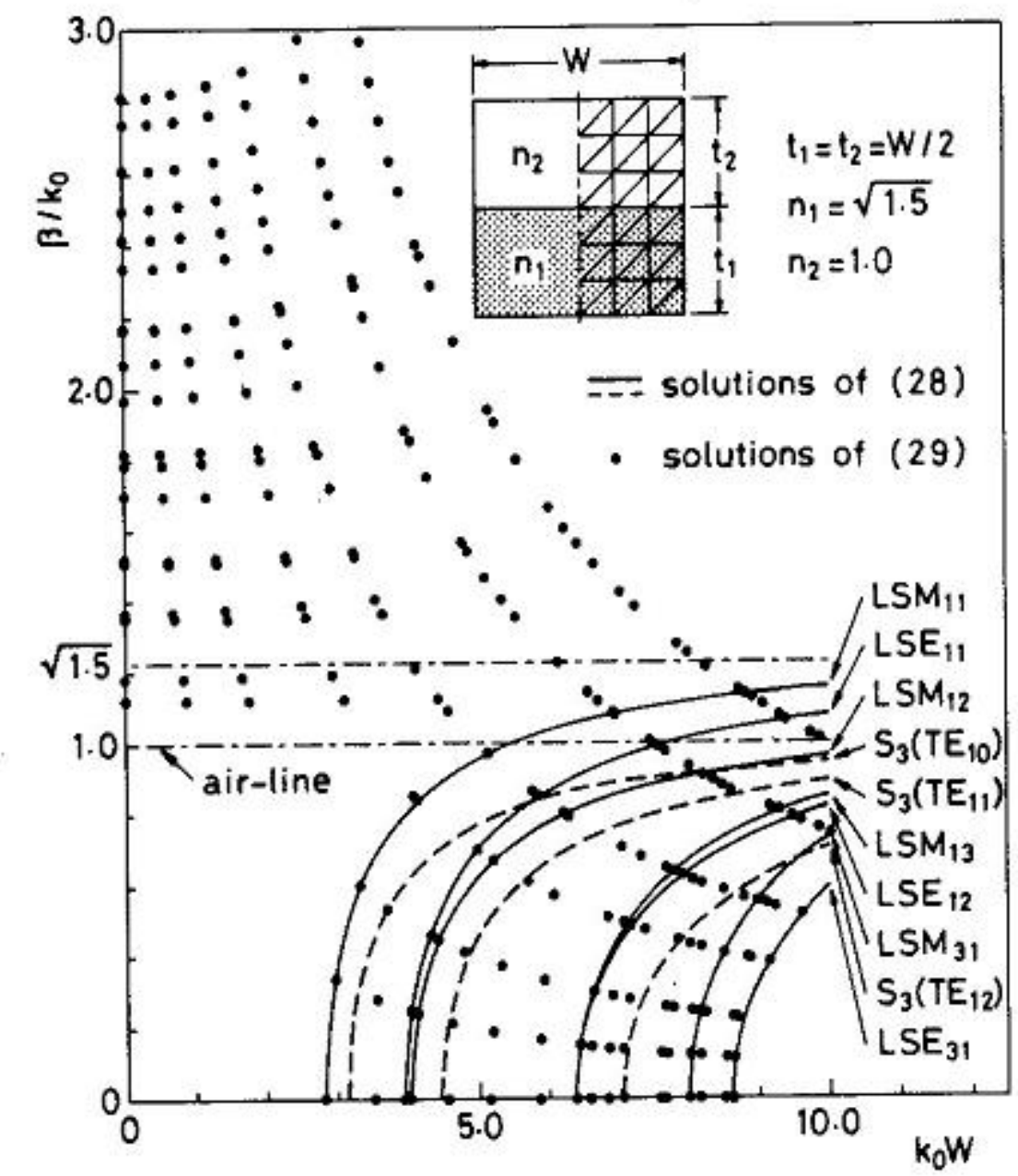

Fig. 2. Solutions of (28) and (29) for a rectangular waveguide half-filled with dielectric of refractive index $\sqrt{1.5}$.

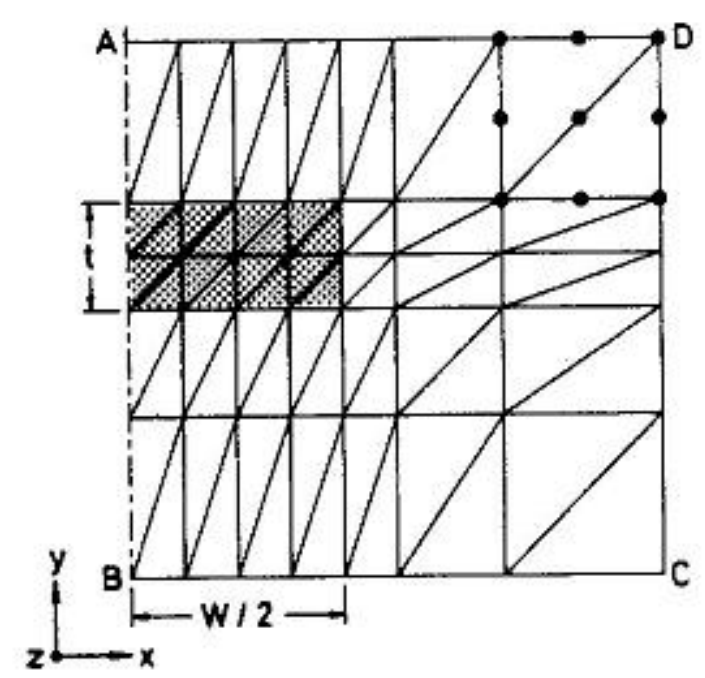

Fig. 3. Finite-element division of an embedded rectangular waveguide and an embossed rectangular waveguide.

represent the solutions of the improved finite-element pro gram in (28), while the solutions of the earlier finite-ele ment program in (29) are indicated by the dots, where the plane of symmetry is assumed to be the perfect magnetic conductor. Computed results (solid lines) for the $L S M_{p q}$ and $L S E_{p q}$ modes [23] agree well with the exact results [23]. Spurious solutions $S_{3}$ (dashed lines) corresponding to the solutions of (14) appear only in the region $\beta / k_{0}<1$. The solutions $S_{3}$ with cutoff frequencies $k_{0} W=\pi, \sqrt{2} \pi$, and $\sqrt{5} \pi$ are equivalent to the $\mathrm{TE}_{10}, \mathrm{TE}_{11}$, and $\mathrm{TE}_{12}$ modes of a "hollow" waveguide of square cross section, respectively. It is found that when (29) is used the spurious solutions are scattered all over the propagation diagram.

Next, let us consider an embedded rectangular wave: guide [4], [5], [10], [12] and an embossed rectangular waveguide [4], [10], [12]. We subdivide one half of the cross section into second-order triangular elements as shown in Fig. 3, where $W$ and $t$ are the width and the thickness of a rectangular core, respectively, and boundaries $B C, C D$, and $D A$ are assumed to be perfect electric conductors. Fig. 4 shows the dispersion characteristics for the $E_{11}^{y}$ mode [21] of these waveguides, where $v=k_{0} t \sqrt{n_{1}^{2}-n_{2}^{2}} / \pi$ and $b=$ 


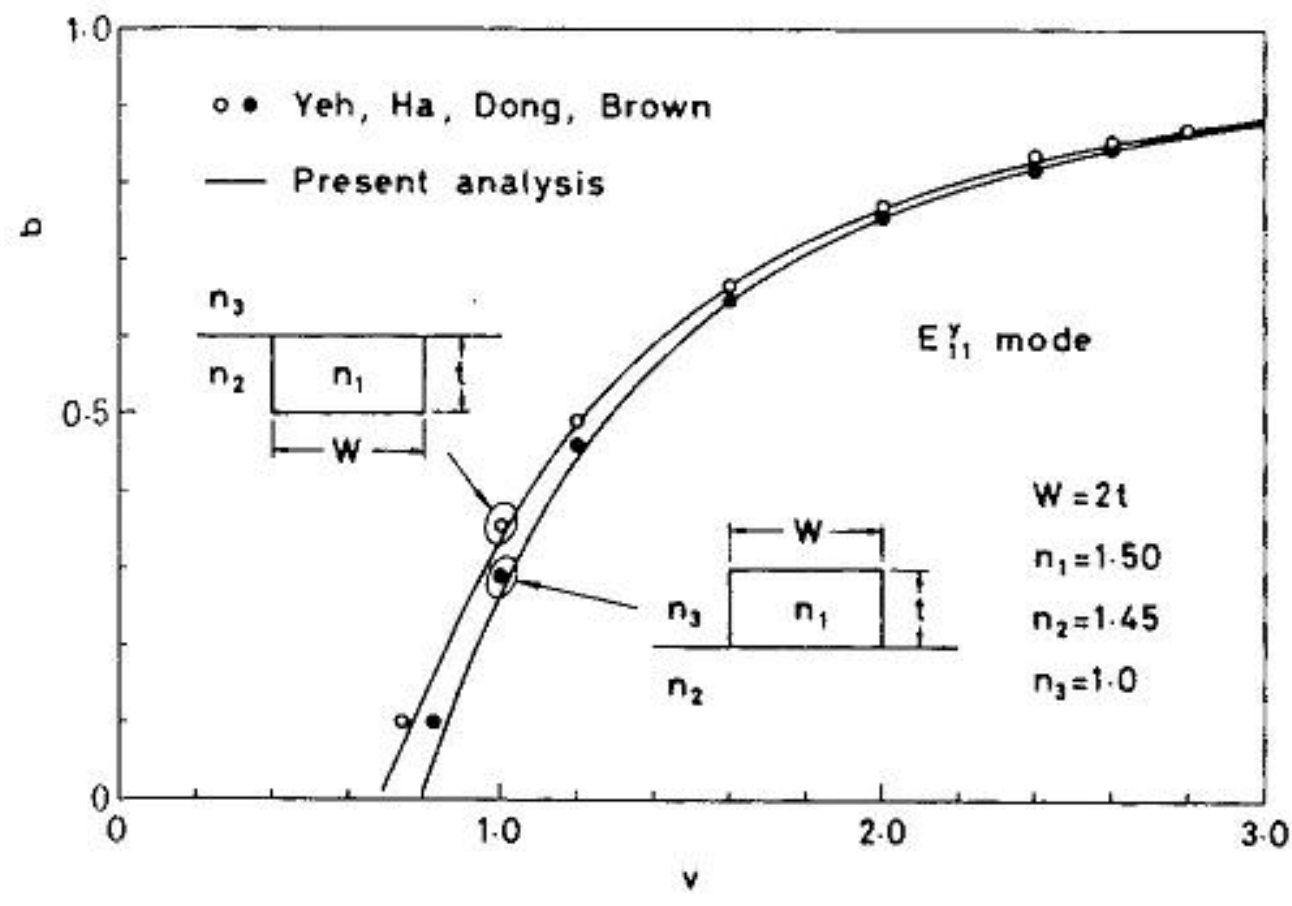

Fig. 4. Dispersion characteristics of an embedded rectangular waveguide and an embossed rectangular waveguide.

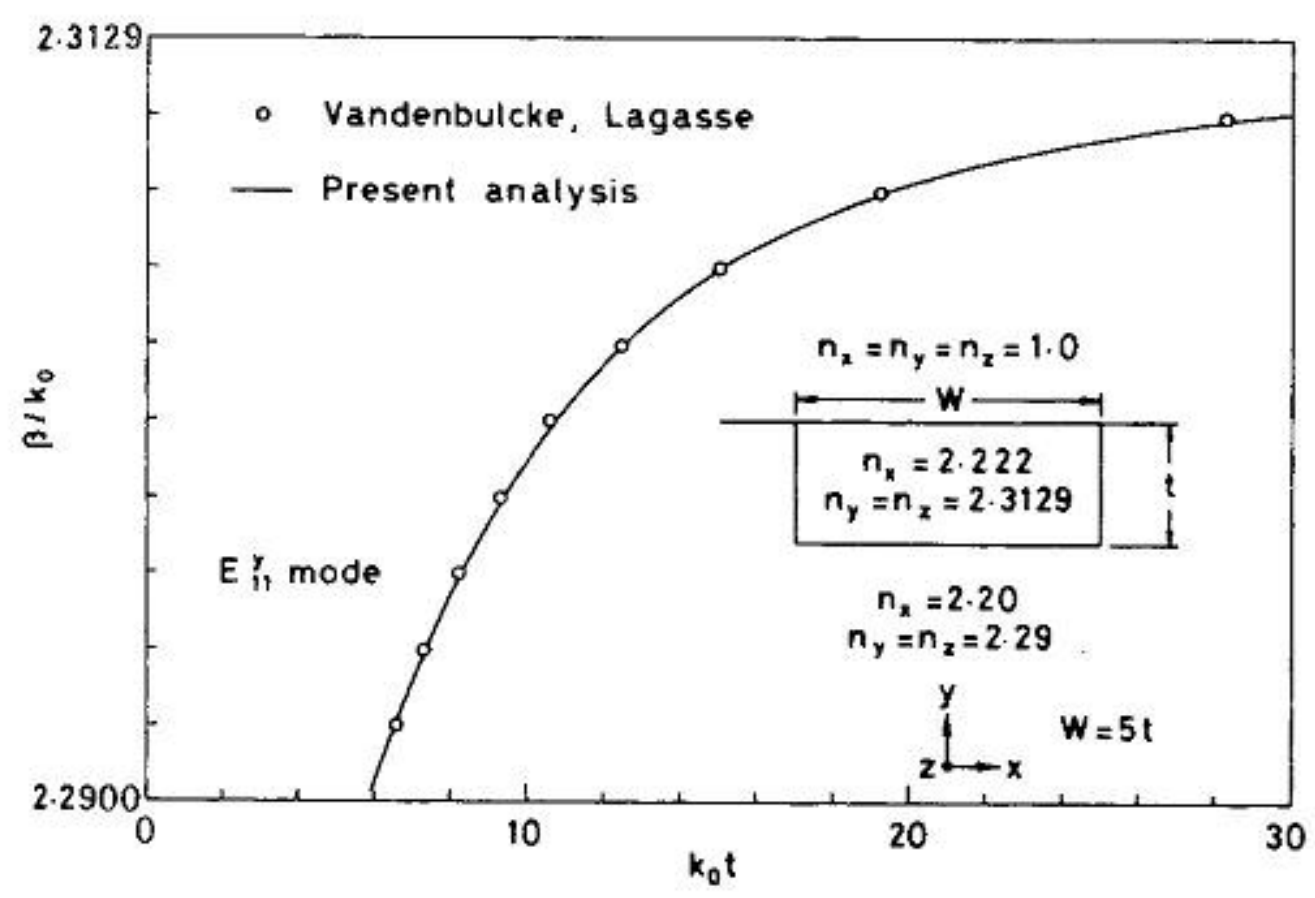

Fig. 5. Dispersion characteristics of an anisotropic embedded rectangular waveguide.

$\left[\left(\beta / k_{0}\right)^{2}-n_{2}^{2}\right] /\left(n_{1}^{2}-n_{2}^{2}\right)$. For the $E_{11}^{y}$ mode, the boundary $A B$ in Fig. 3 (plane of symmetry) becomes the perfect magnetic conductor. Our results agree well with the finiteelement solutions [10] in the $E_{z}-H_{z}$ formulation. Fig. 5 shows the dispersion characteristics for the $E_{11}^{y}$ mode of an anisotropic embedded rectangular waveguide. Our results agree well with the finite-element solutions [5] in the $E_{z}-$ $H_{z}$ formulation. Note that the spurious solutions are included in the solutions of the finite-element method in the $E_{z}-H_{z}$ formulation and they cannot be eliminated mathematically [1]-[14]. The $E_{z}-H_{z}$ formulation contains mathernatical singularities, and the actual solutions are plotted as a continuous interpolated curve between points sufficiently removed from the singularity to be unaffected by it [2], [3], [8], [9], [11]. In order to avoid confusion, such spurious solutions in the $E_{z}-H_{z}$ formulation are not shown in Figs. 4 and 5.

Lastly, let us consider a dielectric square waveguide [4], [21], [22] with four planes of symmetry. We subdivide one quarter or one eighth of the cross section into second-order triangular elements as shown in Fig. 6, where boundaries $C D$ and $D A$ are assumed to be perfect electric conductors and the conditions on boundaries $A B, B C$, and $D B$ (planes of symmetry) are given in Table I. Fig. 7 shows the dispersion characteristics for the $E_{p q}^{x, y}$ modes [21] of this
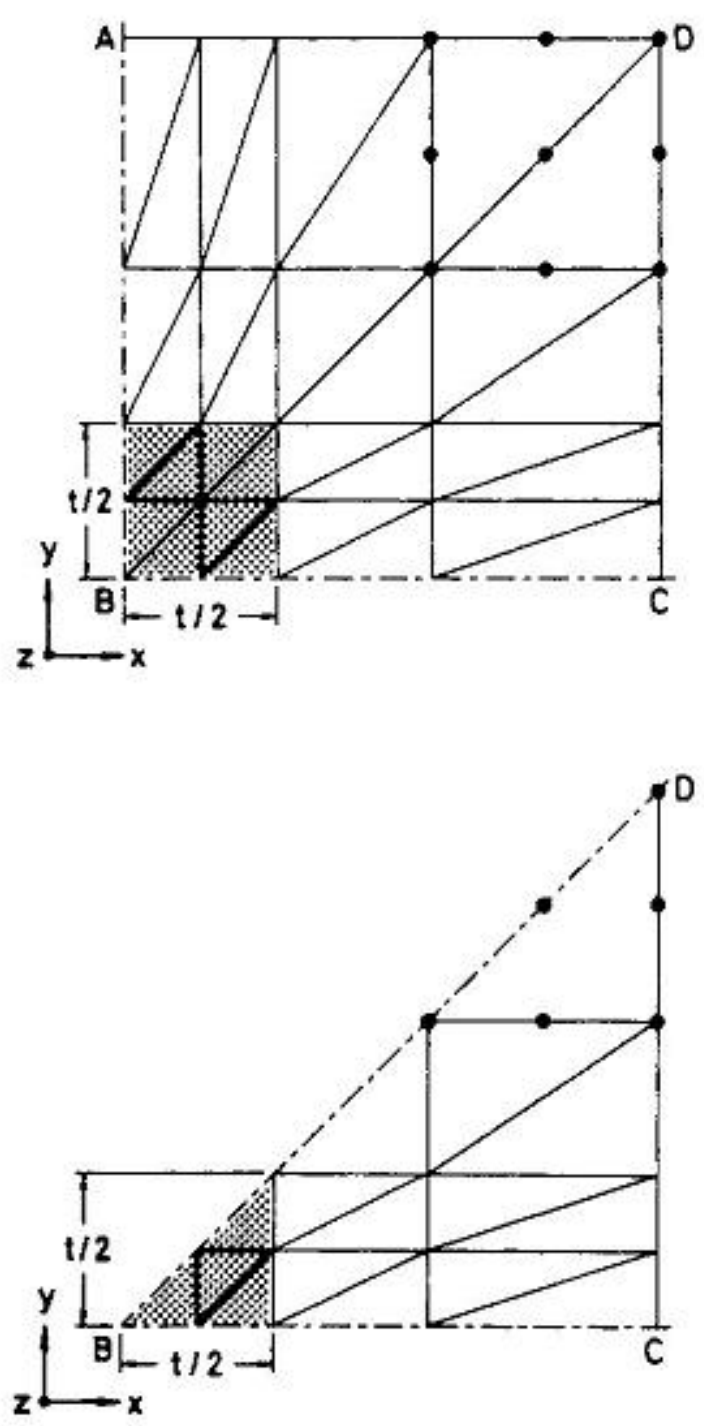

Fig. 6. Finite-element division of a dielectric square waveguide.

TABLE I

Boundary Conditions for a Dielectric Square Waveguide

\begin{tabular}{|c|c|c|c|c|}
\hline \multicolumn{2}{|c|}{ Boundaries } & $A B$ & $\mathrm{BC}$ & DB \\
\hline \multicolumn{2}{|c|}{$\theta$} & $180^{\circ}$ & $270^{\circ}$ & $135^{\circ}$ \\
\hline \multirow{2}{*}{$\begin{array}{l}\text { Boundary } \\
\text { conditions }\end{array}$} & $\begin{aligned} \text { E: } & \text { perfect } \\
& \text { electric } \\
& \text { conductor }\end{aligned}$ & $H_{x}=0$ & $\mathrm{H}_{y}=0$ & $\mathrm{H}_{X}=\mathrm{H}_{Y}$ \\
\hline & $\begin{array}{l}\text { M: perfect } \\
\text { magnetic } \\
\text { conductor }\end{array}$ & $\begin{array}{l}\mathrm{H}_{\mathrm{y}}=0 \\
\mathrm{H}_{\mathrm{z}}=0\end{array}$ & $\begin{array}{l}H_{x}=0 \\
H_{z}=0\end{array}$ & $\begin{aligned} H_{x} & =-H_{y} \\
H_{z} & =0\end{aligned}$ \\
\hline \multirow{6}{*}{ Modes } & $\begin{array}{l}\mathrm{E}_{\text {odd, odd }}^{\mathrm{X}} \\
\mathrm{E}_{\text {even, even }}^{\mathrm{y}}\end{array}$ & E & M & - \\
\hline & $\begin{array}{l}\mathrm{E}_{\text {even, even }}^{\mathrm{X}} \\
\mathrm{E}_{\text {odd, odd }}^{\mathrm{Y}}\end{array}$ & M & E & - \\
\hline & $\mathrm{E}_{\text {even, odd }}^{\mathrm{X}}$ & M & M & M \\
\hline & $E_{\text {odd, even }}^{Y}$ & M & M & $\mathrm{E}$ \\
\hline & $\mathrm{E}_{\text {odd, even }}^{\mathrm{x}}$ & E & $E$ & M \\
\hline & $E_{\text {even, odd }}^{Y}$ & B & E & E \\
\hline
\end{tabular}

waveguide. Our results agree well with the results of the collocation method [22]. For the $E_{21}^{x, y}$ and $E_{12}^{x, y}$ modes whose fields satisfy the boundary condition for the perfect electric or magnetic conductor on the boundary $D B$, the results of Fig. 6(b) are identical to those of Fig. 6(a). This fact proves the validity of (32) and (35). The strength of this approach to boundary conditions is not just the economical use of computer memory but the elimination of spurious solutions through rigorous enforcement of boundary conditions as well. The dots in Fig. 8 represent the solutions of the earlier finite-element program in (29), while the results of the improved finite-element program in 


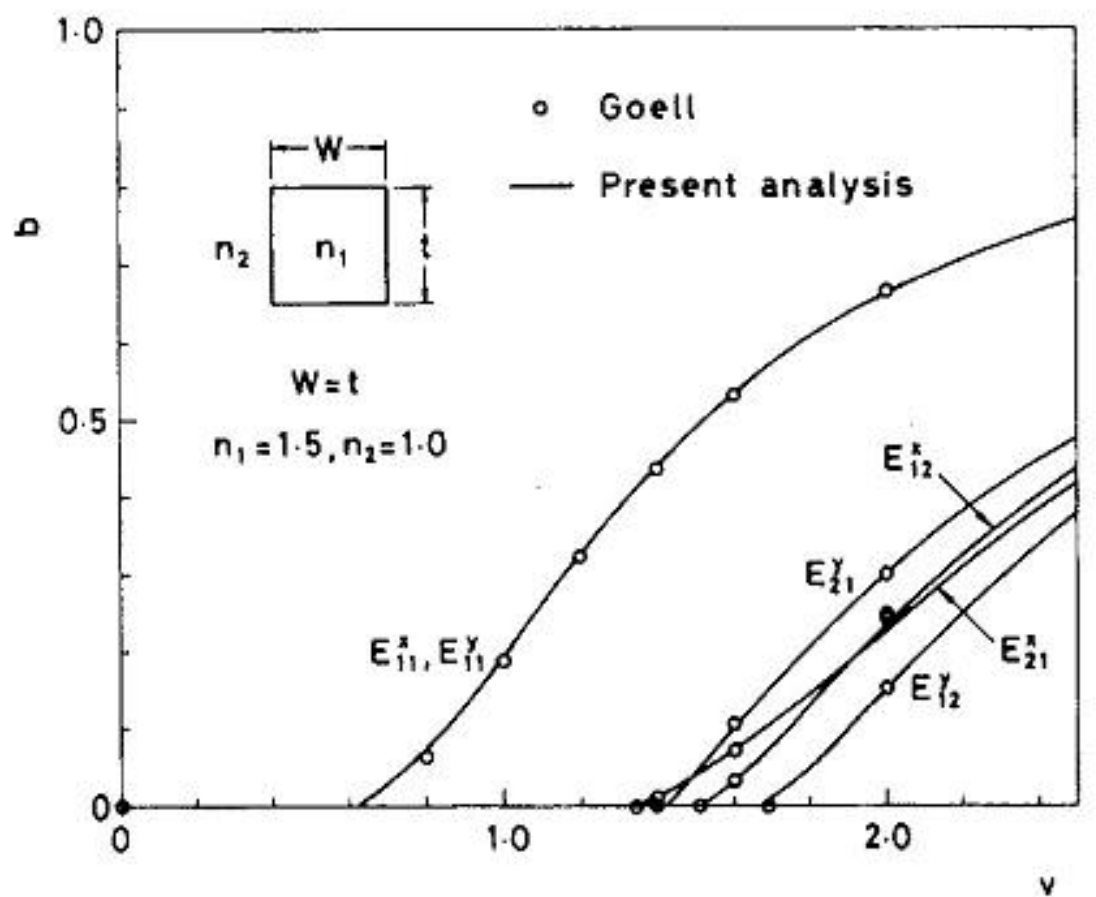

Fig. 7. Dispersion characteristics of a dielectric square waveguide.

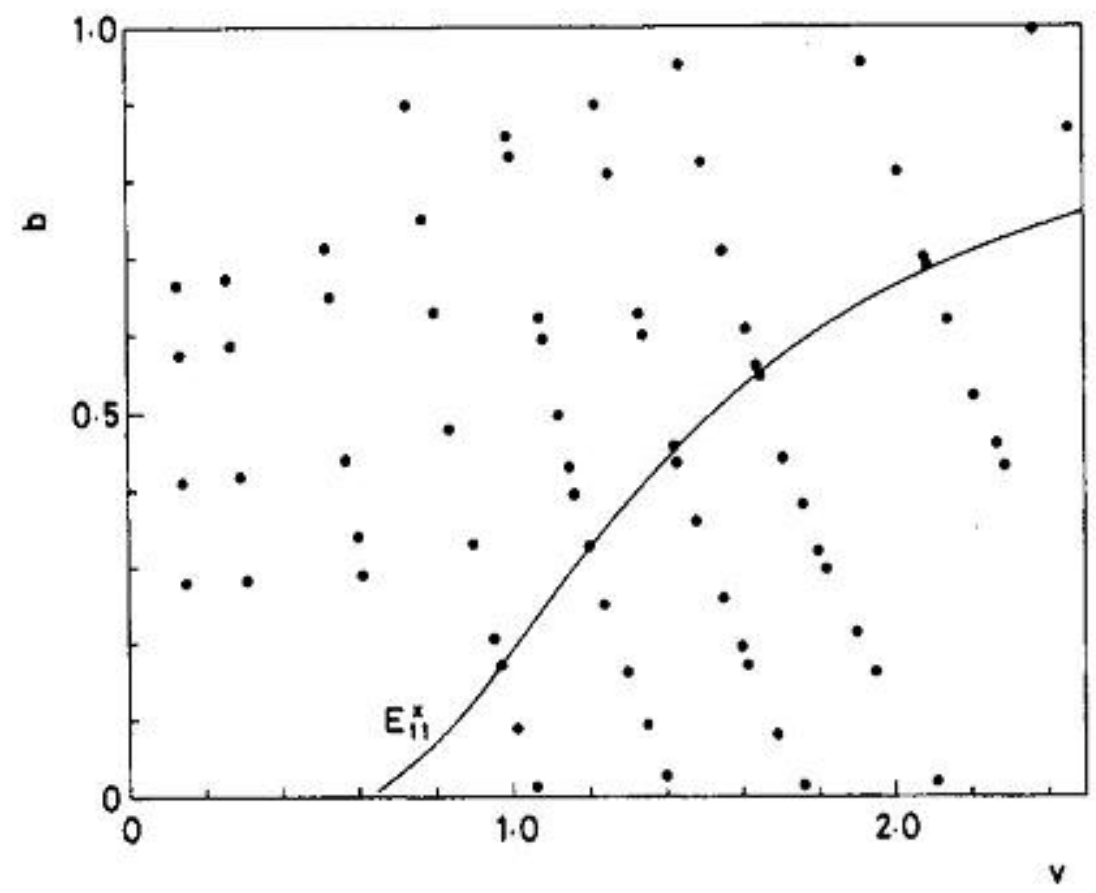

Fig. 8. Solutions of (29) for the waveguide configuration in Fig. ? Boundary conditions are $H_{x}=0$ and $H_{x}=H_{z}=0$ on $A B$ and $B C$ in Fig. 6(a), respectively.

(28) are indicated by a solid line (the $E_{11}^{x}$ mode in Fig. 7), where the conditions on boundaries $A B$ and $B C$ in Fig. 6(a) are $H_{x}=0$ and $H_{x}=H_{z}=0$, respectively. It is found that when (29) is used, numerous spurious solutions appear.

In Figs. 4, 5, and 7, the spurious solutions do not appear because the spurious solutions $\left(S_{3}\right)$ appear only below the "air-line," and the surface-wave modes (the $E_{p q}^{x, y}$ modes) of dielectric waveguides of Figs. 4, 5, and 7 correspond to the solutions above the "air-line."

\section{CONCLUSION}

An improved finite-element method for the analysis of dielectric waveguides with a diagonal permittivity tensor was formulated in terms of all three components of the magnetic field $\boldsymbol{H}$. In this approach, the spurious, nonphysical solutions do not appear anywhere above the "air-line," and therefore the present formulation is very useful for the analysis of the surface-wave modes of dielectric waveguides. The application of this improved finite-element method to the dielectric waveguides with perfect electric and magnetic conductors was also discussed.

This approach can be applied easily to the anisotropic waveguides having a permittivity tensor with nonzero offdiagonal elements.

\section{ACKNOWLEDGMENT}

The authors would like to thank Prof. Y. Kagawa of Toyama University, Dr. M. Ikeuchi of Okawama Univer. sity of Science, and Dr. M. Hano of Yamaguchi University for the interesting and fruitful discussions. They are greatly indebted to the reviewers for their helpful suggestions.

\section{REFERENCES}

[1] S. Ahmed and P. Daly, "Finite-element methods for inhomoge, neous waveguides," Proc. Inst. Elec. Eng., vol. 116, pp. 1661-1664, Oct. 1969.

[2] Z. J. Csendes and P. Silvester, "Numerical solution of dielectric loaded waveguides: I-Finite-element analysis," IEEE Trains. Mi. crowave Theory Tech., vol. MTT-18, pp. 1124-1131, Dec. 1970.

[3] P. Daly, "Hybrid-mode analysis of microstrip by finite-element methods," IEEE Trans. Microwave Theory Tech., vol. MTT-19, pp. 19-25, Jan. 1971.

[4] C. Yeh, S. B. Dong, and W. Oliver, "Arbitrarily shaped inhomogeneous optical fiber or integrated optical waveguides," $J$. Appl. Phys., vol. 46, pp. 2125-2129, May 1975.

[5] P. Vandenbulcke and P. E. Lagasse, "Eigenmode analysis of anisotropic optical fibers or integrated optical waveguides," Electron. Lett., vol. 12, pp. 120-122, Mar. 1977.

[6] A. D. McAuley, "Variational finite-element solution for dissipative waveguides and transportation application," IEEE Trans. Micro. wave Theory Tech., vol. MTT-25, pp. 382-392, May 1977.

[7] T. S. Bird, "Propagation and radiation characteristics of rib waveguide," Electron. Lett., vol. 13, pp. 401-403, July 1977.

[8] M. Ikeuchi, K. Inoue, H. Sawami, and H. Niki, "Spurious solutions in the finite element analysis of microstrip lines," Trans. Inst. Elec. Eng. Japan, vol. 98(A), pp. 415-422, Aug. 1978 (in Japanese).

[9] M. Ikeuchi, K. Inoue, H. Sawami, and H. Niki, "Equivalent circuits for the electromagnetic fields of inhomogeneous dielectric lines by finite element method," Trans. Inst. Electron. Commun. Eng. Japan, vol. J61-B, pp. 888-895, Oct. 1978 (in Japanese).

[10] C. Yeh, K. Ha, S. B. Dong, and W. P. Brown, "Single-mode optical waveguides," Appl. Opt., vol. 18, pp. 1490-1504, May 1979.

[11] M. Ikeuchi, H. Sawami, and H. Niki, "Analysis of open-type dielectric waveguides by the finite-element iterative method," IEEE Trans. Microwave Theory Tech., vol. MTT-29, pp. 234-239, Mar. 1981.

[12] N. Mabaya, P. E. Lagasse, and P. Vandenbulcke, "Finite element analysis of optical waveguides," IEEE Trans. Microwave Theory Tech., vol. MTT-29, pp. 600-605, June 1981.

[13] K. Oyamada and T. Okoshi, "Two-dimensional finite-element calculation of propagation characteristics of axially nonsymmetrical optical fibers," Radio Sci, vol. 17, pp. 109-116, Jan.-Feb. 1982.

[14] M. Aubourg, J. P. Villotte, F. Godon, and Y. Garault, "Finite element analysis of lossy waveguides-Application to microstrip lines on semiconductor substrate," IEEE Trans. Microwave Theory Tech., vol. MTT-31, pp. 326-331, Apr. 1983.

[15] A. Konrad, "High-order triangular finite elements for electromagnetic waves in anisotropic media," IEEE Trans. Microwave Theory Tech., vol. MTT-25, pp. 353-360, May 1977.

[16] J. B. Davies, F. A. Fernandez, and G. Y. Philippou, "Finite element analysis of all modes in cavities with circular symmetry," IEEE Trans. Microwave Theory Tech., vol. MTT-30, pp. 1975-1980, Nov. 1982.

[17] B. M. A. Rahman and J. B. Davies, "Finite-element analysis of optical and microwave waveguide problems," IEEE Trans. Microwave Theory Tech., vol. MTT-32, pp. 20-28, Jan. 1984.

[18] M. Koshiba, K. Hayata, and M. Suzuki, "Vectorial finite-element formulation without spurious solutions for dielectric waveguides," Tech. Res. Rep. Inst. Electron. Commun. Eng. Japan, vol. 83, no. 70, MW83-28, pp. 85-92, June 1983 (in Japanese).

[19] M. Hara, T. Wada, T. Fukusawa, and F. Kikuchi, "A three dimensional analysis of RF electromagnetic fields by the finite element method," IEEE Trans. Magn., vol. MAG-19, pp. 2417-2420, Nov. 1983.

[20] M. Koshiba, K. Hayata, and M. Suzuki, "Vectorial finite-element method without spurious solutions for dielectric waveguide problems," Electron. Lett., vol. 20, pp. 409-410, May 1984.

[21] E. A. J. Marcatili, "Dielectric rectangular waveguide and directional coupler for integrated optics," Bell Syst. Tech. J., vol. 48, pp. 2017-2102, Sept. 1969. 
[22] J. E. Goell, "A circular-harmonic computer analysis of rectangular dielectric waveguides,” Bell Syst. Tech. J., vol. 48, pp. 2133-2160, Sept. 1969.

[23] N. Marcuvitz, Waveguide Handbook. New York: McGraw-Hill, 1951.

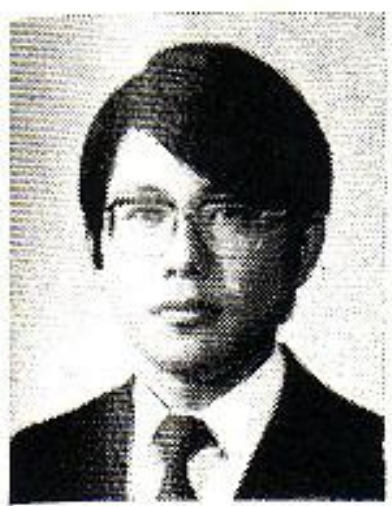

Masanori Koshiba (SM'84) was born in Sapporo, Japan, on November 23, 1948. He received the B.S., M.S., and Ph.D. degrees in electronic engineering from Hokkaido University, Sapporo, Japan, in 1971, 1973, and 1976, respectively.

In 1976, he joined the Department of Electronic Engineering, Kitami Institute of Technology, Kitami, Japan. Since 1979, he has been an Assistant Professor of Electronic Engineering at Hokkaido University. He has been engaged in research on surface acoustic waves, dielectric optical waveguides, and applications of finite-element and boundary element methods to field problems.

Dr. Koshiba is a member of the Institute of Electronics and Communication Engineers of Japan, the Institute of Electrical Engineers of Japan, and the Institute of Television Engineers of Japan.

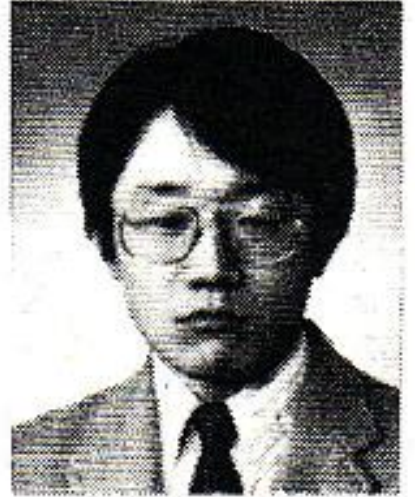

Kazuya Hayata was born in Kushiro, Japan, on December 1,1959 . He received the B.S. and M.S. degrees in electronic engineering from Hokkaido University, Sapporo, Japan, in 1982 and 1984, respectively.

Since 1984, he has been a Research Assistant of Electronic Engineering at Hokkaido University. He has been engaged in research on dielectric optical waveguides and surface acoustic waves.

Mr. Hayata is a member of the Institute of Electronics and Communication Engineers of Japan.

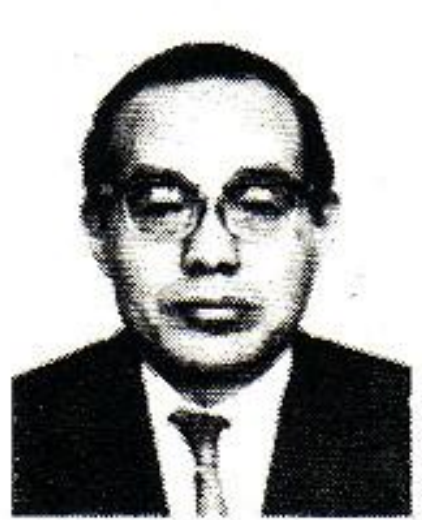

Michio Suzuki (SM'57) was born in Sapporo, Japan, on November 14, 1923. He received the B.S. and Ph.D. degrees in electrical engineering from Hokkaido University, Sapporo, Japan, in 1946 and 1960 , respectively.

From 1948 to 1962 , he was an Assistant Professor of Electrical Engineering at Hokkaido University. Since 1962, he has been a Professor of Electronic Engineering at Hokkaido University. From 1956 to 1957 , he was a Research Associate at the Microwave Research Institute of Polytechnic Institute of Brooklyn, Brooklyn, NY.

Dr. Suzuki is a member of the Institute of Electronics and Communication Engineers of Japan, the Institute of Electrical Engineers of Japan, and the Institute of Television Engineers of Japan. 